\title{
3D PULSE DIFFRACTION ON A PHASE-SHIFTED BRAGG GRATING
}

\author{
N.V. Golovastikov, D.A. Bykov, L.L. Doskolovich \\ Image Processing Systems Institute - Branch of the Federal Scientific Research Centre \\ "Crystallography and Photonics" of Russian Academy ofSciences, Samara, Russia, \\ Samara National Research University, Samara, Russia
}

\begin{abstract}
We consider diffraction of a three-dimensional (3D) spatiotemporal optical pulse by a phase-shifted Bragg grating. Resonant approximations of the reflection and transmission coefficients of a phase-shifted Bragg grating as functions of the angular frequency and the in-plane components of the wave vector are obtained. Using these approximations, analytical expressions for reflected and transmitted 3D pulse envelopes are derived. The presented rigorous simulation results fully confirm the proposed theoretical description.
\end{abstract}

Keywords: Bragg grating, resonance, all-optical information processing.

Citation: Golovastikov NV, Bykov DA, Doskolovich LL. 3D pulse diffraction on a phase-shifted Bragg grating. CEUR Workshop Proceedings, 2016; 1638: 32-38. DOI: 10.18287/1613-0073-2016-1638-32-38

\section{Introduction}

Optical devices implementing required temporal and spatial transformations of optical signals are of great interest for a wide range of practical applications including alloptical information processing and analog optical computing [1].Various resonant structures are known to implement these operations including phase-shifted Bragg gratings (PSBG). PSBGs have been used both for temporal differentiation and integration of an optical pulse envelope [2-4] and for spatial differentiation and integration of a monochromatic optical beam profile $[5,6]$. The utilization of resonant structures is attributable to the fact that their reflection (transmission) coefficient of the structure in the vicinity of the resonance can be described by the Fano profile, which in turn can approximate the transfer function of a differentiating or integrating filter. In case of a PSBG this resonant effect is caused by the excitation of the structure's eigenmode localized at the defect layer.

In the previous works, temporal and spatial transformations of the incident pulse envelope were considered separately. Temporal transformations in [2-4] were investigated without taking into account the spatial configuration of the incident optical pulse, whereas spatial operations in $[5,6]$ were studied in the case of a monochromatic incident beam. In this regard, the description of spatiotemporal transformations 
of optical pulses implemented by PSBG in the general 3D case is of great interest. Recently published results $[7,8]$ concerned 2D spatiotemporal optical pulse diffraction by a resonant grating.

In this work, a theoretical description of the 3D optical pulse diffraction by a PSBG is presented for the first time. Resonant approximations of the PSBG's reflection and transmission coefficients as functions of the angular frequency and the in-plane wave vector component are obtained. It is shown that at normal incidence the transformation of the pulse envelope can be described by a hyperbolic partial differential equation. The proposed theoretical description is confirmed by rigorous simulation results.

\section{3D pulse envelope}

Consider normal incidence of a 3D optical pulse on a multilayer structure (Fig. 1). It can be shown that reflected and transmitted pulse envelopes $u_{R, T}(x, y, t)$ correspond to theincident pulse envelope $v(x, y, t)$ passing through a linear system. The connection between the Fourier spectrum of the incident pulse $V\left(k_{x}, k_{y}, \omega\right)$ and the Fourier spectrum of the reflected (transmitted) pulse $U_{R, T}\left(k_{x}, k_{y}, \omega\right)$ has the form:

$U_{R, T}\left(k_{x}, k_{y}, \omega\right)=H_{R, T}\left(k_{x}, k_{y}, \omega\right) V\left(k_{x}, k_{y}, \omega\right)$,

where $H_{R, T}\left(k_{x}, k_{y}, \omega\right)$ is the linear system's transfer function (TF), expressed through the structure's reflection (transmission) coefficient:

$$
\begin{aligned}
& H_{R}\left(k_{x}, k_{y}, \omega\right)=R\left(\sqrt{k_{x}^{2}+k_{y}^{2}}, \omega+\omega_{0}\right), \\
& H_{T}\left(k_{x}, k_{y}, \omega\right)=T\left(\sqrt{k_{x}^{2}+k_{y}^{2}}, \omega+\omega_{0}\right) .
\end{aligned}
$$

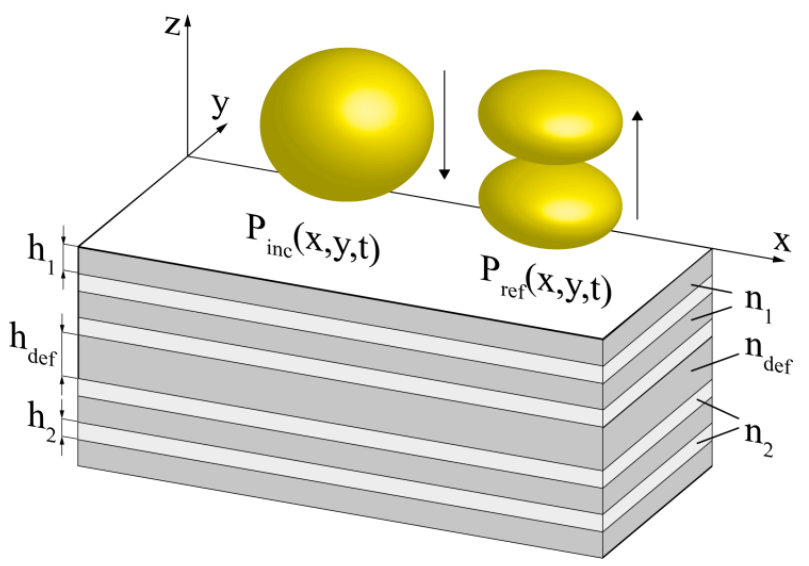

Fig.1. 3D pulse diffraction by a PSBG 
Here $\omega_{0}$ is the central frequency of the pulse, $k_{x}, k_{y}$ are components of the in-plane wave vector. Note that TFs (2) are functions of angular frequency and in-plain wave vector components. In this regard, these TFs are generalizations of TFs which describe temporal transformations of an optical pulse [4] and spatial transformations of an optical beam [5].

We assume that the incident pulse is polarized along the $x$ axis at $z=0$. This means that the incident pulse envelope $v(x, y, t)$ corresponds to the $E_{x}$ component of the electric field $\left(E_{y} \equiv 0\right)$, and the reflection and transmission coefficients $R\left(k_{x}, k_{y}, \omega+\omega_{0}\right)$ and $T\left(k_{x}, k_{y}, \omega+\omega_{0}\right)$ in (2) are expressed through the reflection and transmission coefficients of TE- and TM-polarized plane waves incident on the structure.

\section{Envelope transformation}

To analytically describe transformations of an optical pulse envelope upon diffraction by a PSBG (1) we derived resonant approximation for the grating's reflection and transmission coefficients. This approximation holds true in the vicinity of normal incidence $\left(k_{x}=k_{y}=0\right)$ and the resonance frequency corresponding to the mode of the defect layer $\omega_{p}$ and has the following form:

$$
\begin{aligned}
& R\left(k_{x}, k_{y}, \omega\right)=\gamma_{R} \frac{v_{g}^{2}\left(k_{x}^{2}+k_{y}^{2}\right)-\left(\omega-\omega_{z}^{R}\right)\left(\omega+\omega_{z}^{R}\right)}{v_{g}^{2}\left(k_{x}^{2}+k_{y}^{2}\right)-\left(\omega-\omega_{p}\right)\left(\omega+\omega_{p}^{*}\right)}, \\
& T\left(k_{x}, k_{y}, \omega\right)=\gamma_{T} \frac{v_{g}^{2}\left(k_{x}^{2}+k_{y}^{2}\right)-\left(\omega-\omega_{z}^{T}\right)\left(\omega+\omega_{z}^{T *}\right)}{v_{g}^{2}\left(k_{x}^{2}+k_{y}^{2}\right)-\left(\omega-\omega_{p}\right)\left(\omega+\omega_{p}^{*}\right)},
\end{aligned}
$$

where $v_{g}^{2} \in \mathbf{R}$ corresponds to the group velocity of an eigenmode of the structure; $\gamma_{R}$ $\gamma_{T}$ are the non-resonant (far-from-resonance) reflection and transmission coefficients. Representations (3) can be considered as a generalization of reflection and transmission coefficients resonant approximations to a three-dimensional case. At a fixed frequency $\omega$ equations (3) become the known approximations of the reflection and transmission coefficients as functions of $k_{x}$ in the vicinity of normal incidence $\left(k_{x}=k_{y}=0\right)$ [5]. At $k_{x}=k_{y}=0$ the spectra (3) are reduced to the conventional Fano profile [4]. Note that expressions (3) generalize the previously proposed resonant approximations of PSBG spectra [9] by accounting for the second pole of the resonant structure with negative real part $-\omega_{p}^{*}$.

Taking into account (3), we rewrite TFs (2) as product of two TFs, corresponding to transformations implemented by the numerator $\left(H_{R, T}^{1}\left(k_{x}, k_{y}, \omega\right)\right)$ and denominator $\left(H_{R, T}^{2}\left(k_{x}, k_{y}, \omega\right)\right)$ of the original TF $H_{R, T}\left(k_{x}, k_{y}, \omega\right)$ : 


$$
\begin{aligned}
& H_{R, T}\left(k_{x}, k_{y}, \omega\right)=H_{R, T}^{1}\left(k_{x}, k_{y}, \omega\right) \cdot H_{R, T}^{2}\left(k_{x}, k_{y}, \omega\right)= \\
& =\gamma_{R, T}\left(v_{g}^{2}\left(k_{x}^{2}+k_{y}^{2}\right)-\left(\omega+\omega_{0}-\omega_{z}^{R, T}\right)\left(\omega+\omega_{0}+\omega_{z}^{R, T^{*}}\right)\right) . \\
& \cdot \frac{1}{v_{g}^{2}\left(k_{x}^{2}+k_{y}^{2}\right)-\left(\omega+\omega_{0}-\omega_{p}\right)\left(\omega+\omega_{0}+\omega_{p}^{*}\right)}
\end{aligned}
$$

Applying the inverse Fourier transform to Eq. (1) and taking into account Eq. (4), we obtain the following differential equation for the envelope of the reflected (transmitted) pulse $u_{R, T}(x, y, t)$ :

$$
\begin{array}{r}
\frac{\partial^{2} u_{R, T}}{\partial t^{2}}-v_{g}^{2} \Delta u_{R, T}-2 \mathrm{i}\left(\omega_{0}-\mathrm{i} \operatorname{Im}\left[\omega_{p}\right]\right) \frac{\partial u_{R, T}}{\partial t}+ \\
+\left(\omega_{0}-\omega_{p}\right)\left(\omega_{0}+\omega_{p}^{*}\right) u_{R, T}=f_{R, T},
\end{array}
$$

where

$$
\begin{aligned}
f_{R, T}(x, y, t) & =\gamma_{R, T}\left(\frac{\partial^{2} v}{\partial t^{2}}-v_{g}^{2} \Delta v-2 \mathrm{i}\left(\omega_{0}-\mathrm{i} \operatorname{Im}\left[\omega_{z}^{R, T}\right]\right) \frac{\partial v}{\partial t}+\right. \\
& \left.+\left(\omega_{0}-\omega_{z}^{R, T}\right)\left(\omega_{0}+\omega_{z}^{R, T^{*}}\right) v\right),
\end{aligned}
$$

$\Delta=\partial^{2} / \partial x^{2}+\partial^{2} / \partial y^{2}$ is the Laplace operator with respect to the spatial coordinates.

Note that for low-quality-factor resonances, the function $H_{R, T}^{2}\left(k_{x}, k_{y}, \omega\right)$ in Eq.(4) is slowly varying. That means that in this case the incident pulse undergoes the transformation $f_{R, T}(x, y, t)$ (6) corresponding to the linear system with the TF $H_{R, T}^{1}\left(k_{x}, k_{y}, \omega\right)$ [8]. Equation(6) demonstrates that the PSBG enables the optical implementation of several important differential operators including the computation of the Laplacian with respect to spatial coordinates (the second term in Eq. (6)) and the computation of the first temporal derivative (the third term in $\mathrm{Eq}(6)$ ). These operations can be performed in reflection for a pulse with central frequency $\omega_{0}=\omega_{z}^{R}$ $\left(\operatorname{Im}\left[\omega_{z}^{R}\right]=0\right)[7,8]$. Note that in the previous works these operations were studied separately for monochromatic beams and infinitely wide pulses respectively. In this regard equations (5), (6)generalize spatial and temporal transformations of optical signals analyzed in $[3,5]$.

The solution to the differential equation (5)can be obtained in the following form:

$$
\begin{aligned}
& u_{R, T}(x, y, t)=\int_{-\infty}^{+\infty} \int_{-\infty}^{+\infty} \int_{-\infty}^{+\infty} f_{R, T}(\xi, \eta, \tau) \cdot h_{3 \mathrm{D}}(x-\xi, y-\eta, t-\tau) \mathrm{d} \xi \mathrm{d} \eta \mathrm{d} \tau, \\
& h_{3 \mathrm{D}}(x, y, t)= \begin{cases}\exp \left\{\mathrm{i} \omega_{0} t+\operatorname{Im}\left[\omega_{p}\right] t\right\} \frac{\cos \left(\operatorname{Re}\left[\omega_{p}\right] \sqrt{t^{2}-\left(x^{2}+y^{2}\right) / v_{g}^{2}}\right)}{2 \pi v_{g}^{2} \sqrt{t^{2}-\left(x^{2}+y^{2}\right) / v_{g}^{2}}}, & x^{2}+y^{2} \leq v_{g}^{2} t^{2}, \\
0, & x^{2}+y^{2} \geq v_{g}^{2} t^{2},\end{cases}
\end{aligned}
$$


Note that $h_{3 \mathrm{D}}(x, y, t)$ is the fundamental solution of (5) and the impulse response function of the linear system with TF $H_{R, T}^{2}\left(k_{x}, k_{y}, \omega\right)(4)$. The obtained solution has a clear physical interpretation. The integration in Eq. (7) is performed over the area located inside the cone $x^{2}+y^{2}<v_{g}^{2} t^{2}$. This area can be considered as an analogue of the light cone: it includes the points, the light from which can reach the considered point in a time $t$. Assuming that the group velocity of the eigenmode of the structure $v_{g}$ does not exceed the speed of light, the impulse response function $h_{3 \mathrm{D}}(x, y, t)$ satisfies the relativistic causality condition.

\section{$4 \quad$ Numerical simulations}

To confirm the accuracy of the obtained model we numerically simulated diffraction of an optical pulse on a PSBG with high quality-factor resonance (29 layers, quality-factor $\left.Q=\operatorname{Re}\left[\omega_{p}\right] /\left(-2 \operatorname{Im}\left[\omega_{p}\right]\right)=7848.6\right)$. The following values of the refractive indices of the BG layers, defect layers, superstrate and substrate were used in the simulations: $n_{1}=2.4547\left(\mathrm{TiO}_{2}\right), n_{2}=1.4446\left(\mathrm{SiO}_{2}\right), n_{\text {def }}=n_{1}, n_{\text {sup }}=n_{\text {sub }}=1$. The thicknesses of the layers were calculated so that the grating has zero reflection for Bragg wavelength $\lambda_{B}=1500 \mathrm{~nm}$ and the incidence angle $\theta_{0}=0$. Fig. 2a shows the PSBG transmission spectrum $\left|T\left(k_{x}, \omega\right)\right|$ calculated using RCWA in the case of TM-polarization (left half, $k_{x}<0$ ) and calculated using the resonant approximation (3) (right half, $\left.k_{x}>0\right)$. A sharp transmission maximum caused by the excitation of an eigenmode in the defect layer is evident in Fig 2a. The complex frequency of the eigenmode $\omega_{p}=1255.77-0.08 \mathrm{ips}^{-1}$ was found as a pole of the structure's scattering matrix at normal incidence $\left(k_{x}=k_{y}=0\right)$. The rest of the approximation parameters $\omega_{z}^{T}=966.84+801.35 \mathrm{i} \mathrm{ss}^{-1}, \gamma_{T}=0.99 \cdot 10^{-4}$ and $v_{g}=159 \mu \mathrm{m} / \mathrm{ps}$ - were obtained using an optimization procedure aimed at minimizing the difference between the transmission coefficient calculated using RCWA and the approximate expression (3) at several characteristic points of the spectrum. The normalized root-mean-square deviation (NRMSD) of the spectra in Fig. 2a amounts to 2.1\%. The NRMSD amounts to $2.0 \%$ for the considered example in the case of TE-polarization. Resonant representation (3) provides similar accuracy also when used for the approximation of the reflection spectra.

We simulated the diffraction of an incident Gaussian pulse $v(x, y, t)=\exp \left\{-\left(x^{2}+y^{2}\right) / \sigma^{2}-t^{2} / \sigma_{t}^{2}\right\}$ with the following parameters: $\sigma_{t}=1 \mathrm{ps}$, $\sigma=2 \mu \mathrm{m}$, central frequency $\omega_{0}=2 \pi c / \lambda_{\mathrm{B}}=1255.77 \mathrm{ps}^{-1}$ (fig. $2 \mathrm{~b}$ ) by the considered grating. Figure 3(b) compares the envelopes of the transmitted pulse calculated using RCWA $(x<0)$ and using the analytical model (7), (8) $(x>0)$. The NRMSD of the 
distributions in Fig. 2c amounts to $2.2 \%$, which confirms high accuracy of the proposed model.

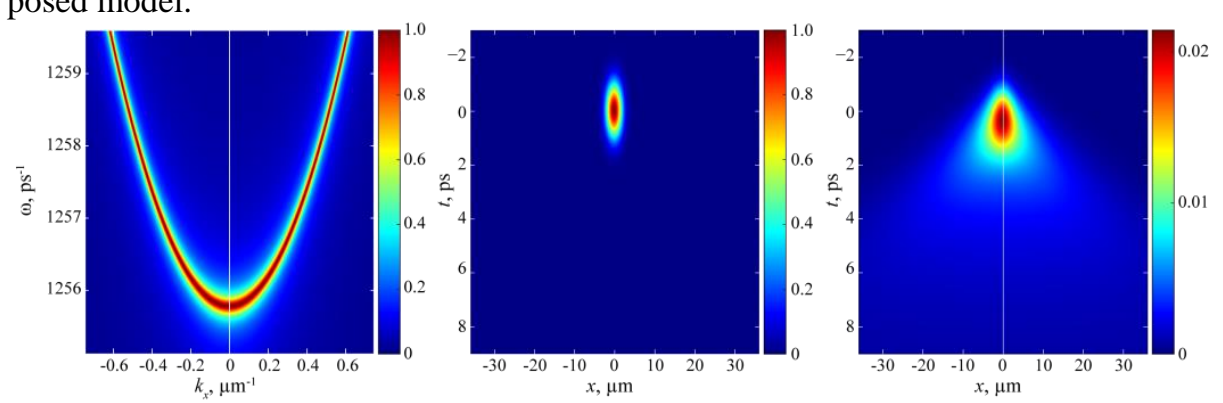

Fig.2. (a) Transmission spectrum $\left|T\left(k_{x}, k_{y}=0, \omega\right)\right|$, (b) incident pulse envelope

$\left|P_{\text {inc }}(x, y=0, t)\right|,(\mathrm{c})$ transmitted pulse envelope $\left|P_{\mathrm{tr}}(x, y=0, t)\right|$

Let us note that the computational cost of the PSBG spectra estimation (Eq.(3)), and the solution of the 3D pulse diffraction problem using Eqs.(7) is much smaller comparing to the direct solution of Maxwell's equations using the RCWA technique. Indeed, for the calculation of the resonant approximations parameters (3) or the convolution kernel (8) it is sufficient to rigorously solve the diffraction problem only for several points in the $\left(k_{x}, k_{y}, \omega\right)$ space. Moreover, once calculated parameters of the convolution kernel (8) allow one to simulate the diffraction of an arbitrary pulse.

\section{Conclusion}

We have proposed resonant approximations of the reflection and transmission coefficients of the PSBG as functions of the angular frequency and the in-plane component of the wave vector. On the basis of the proposed approximations, we obtained theoretical description of the class of the spatiotemporal transformations of a 3D optical pulse envelope upon diffraction by a PSBG. The presented simulation results obtained using the rigorous coupled-wave analysis technique fully confirm the theoretical description. These results may find application in the design of novel planar devices for all-optical information processing and analog optical computing.

\section{Acknowledgments}

This study was funded by Russian Science Foundation (grant no. №14-19-00796).

\section{References}

1. Silva A, Monticone F, Castaldi G, Galdi V, Alù A, Engheta N. Performing Mathematical Operations with Metamaterials. Science, 2014; 343(6167): 160-163. 
2. Rivas LM, Boudreau S, Park Y, Slavík R, LaRochelle S, Carballar A, Azaña J. Experimental demonstration of ultrafast all-fiber high-order photonic temporal differentiators. Opt. Lett., 2009; 34(12): 1792-1794.

3. Kulishov M, Azaña J. Design of high-order all-optical temporal differentiators based on multiple-phase-shifted fiber Bragg gratings. Optics Express, 2007; 15(10): 6152-6166.

4. Nam Quoc Ngo. Design of an optical temporal integrator based on a phase-shifted fiber Bragg grating in transmission. Opt. Lett., 2007; 32(20): 3020-3022.

5. Bykov DA, Doskolovich LL. On the diffraction of an optical beam by a phase shifted Bragg grating. Computer Optics, 2014; 38(4): 590-597 [in Russian].

6. Golovastikov NV, Bykov DA, Doskolovich LL. Spatial integration of optical beams using phase-shifted Bragg grating. Computer Optics, 2014; 38(3): 372-376 [in Russian].

7. Golovastikov NV, Bykov DA, Doskolovich LL, Soifer VA. Spatiotemporal optical pulse transformation by a resonant diffraction grating. Journal of Experimental and Theoretical Physics, 2015; 121(5): 785-792.

8. Golovastikov NV, Bykov DA, Doskolovich LL. Spatiotemporal pulse shaping using resonant diffraction gratings. Optics Letters, 2015; 40(15): 3492-3495.

9. Doskolovich LL, Golovastikov NV, Bykov DA, Kharitonov SI. Resonant approximation of phase-shifted bragg grating (PSBG) spectra. Computer Optics, 2015; 39(3):311-318 [in Russian]. DOI: 10.18287/0134-2452-2015-39-3-311-318. 